Int. J. Electrochem. Sci., 15 (2020) 9499 - 9516

\title{
State of Charge (SOC) Estimation of Lithium-ion Battery Based on Adaptive Square Root Unscented Kalman Filter
}

\author{
Wang Kai ${ }^{1, *}$, Feng Xiao ${ }^{1}$, Pang Jinbo ${ }^{2}$, Ren Jun ${ }^{1}$, Duan Chongxiong ${ }^{3}$, Li Liwei ${ }^{1, *}$ \\ ${ }^{1}$ School of Electrical Engineering, Qingdao University, Qingdao, 266071, China \\ ${ }^{2}$ Collaborative Innovation Center of Technology and Equipment for Biological Diagnosis \\ and Therapy in Universities of Shandong, Institute for Advanced Interdisciplinary \\ Research (iAIR), University of Jinan, Shandong, Jinan 250022, China \\ ${ }^{3}$ School of Materials Science and Energy Engineering, Foshan University, Foshan, \\ 528231, China \\ *E-mail: wkwj888@163.com and ytllw@163.com
}

doi: $10.20964 / 2020.09 .84$

Received: 8 May 2020 / Accepted: 19 July 2020 / Published: 10 August 2020

\begin{abstract}
The improved battery management system (BMS) can give full play to the best performance of power battery, and the state of charge (SOC) estimation of power lithium-ion battery is the core and key technology of BMS. The Kalman filter method with the first-order Thevenin model cannot obtain better estimation results because of the limited model precision. Aiming at solving the above problems, this paper presents a second-order Thevenin equivalent circuit model. The idea of the Sage-Husa adaptive algorithm and square root filter is introduced based on the Unscented Kalman Filter (UKF) algorithm. The adaptive square root Unscented Kalman Filter (ASRUKF) algorithm is formed to improve the precision of SOC estimation. Experiments on SOC estimation of the battery are carried out under three different working conditions. The experimental results show that the ASRUKF algorithm under the second-order Thevenin equivalent circuit model can converge quickly and achieve high precision in SOC estimation.
\end{abstract}

Keywords: lithium-ion battery, SOC estimation, equivalent circuit model, adaptive square root Unscented Kalman Filter

\section{$\underline{\text { FULL TEXT }}$}

(C) 2020 The Authors. Published by ESG (www.electrochemsci.org). This article is an open access article distributed under the terms and conditions of the Creative Commons Attribution license (http://creativecommons.org/licenses/by/4.0/). 